УДК 621.396

DOI: $10.15827 / 0236-235 X .126 .290-298$

\title{
Комплексная математическая модель дирижабельного радиолокационного комплекса обнаружения малозаметных воздушных иелей
}

\author{
С.В. Суша 1, начальник отдела, sushasv2008@yandex.ru \\ 1 Управление Минобороны России, г. Тверь, 170100, Россия
}

Одним из этапов создания дирижабельного радиолокационного комплекса обнаружения малозаметных целей и оценки эффективности его функционирования является разработка комплексной математической модели, структура которой представлена в данной статье. Модель разработана в интересах обоснования технического облика комплекса и особенностей его применения, а также для оценки эффективности функционирования и боевых (информационных) возможностей. Разработка комплексной математической модели базируется на основных принципах построения и структуре данного комплекса. Сам комплекс является информационным средством, представляя собой сложную радиотехническую систему. Аппаратно-программные средства комплекса должны размещаться как на земле, так и в воздухе.

Структура комплексной математической модели включает несколько функционально законченных блоков и моделей, в частности, модели фоноцелевой обстановки, Земли, бортовых систем дирижабельного радиолокационного комплекса и др. В статье описываются частные математические модели, входящие в состав комплексной, их взаимосвязь друг с другом.

Модель радиолокационной станции дирижабельного радиолокационного комплекса строится на основе расчетов параметра обнаружения (отношения сигнал/шум) по уравнению радиолокации и является аналитической моделью приемо-передающего тракта и процесса распространения сигналов. Динамика функционирования имитируется путем последовательного изменения ее состояния через некоторые интервалы времени. Модель обеспечивает динамический подыгрыш выходной информации, аналогичной выходной информации моделируемого средства. Кроме того, она предусматривает возможность имитации обмена с системой управления, имеет параметры, управление которыми позволит имитировать управление режимами функционирования моделируемого средства.

Рассмотрены возможности комплексной математической модели дирижабельного радиолокационного комплекса обнаружения малозаметных воздушных целей.

Ключевые слова: комплексная математическая модель, малозаметная воздушная цель, радиолокационная станция, дирижабельный радиолокаџионный комплекс.

В современных условиях наибольшую угрозу национальной безопасности РФ создают группировки средств воздушного нападения. Для воздушных ударов по данным группировкам предполагается применение самолетов стратегической и тактической авиации, малоразмерных (малозаметных) воздушных иелей (МВЦ), как правило, крылатых ракет и беспилотных летательных аппаратов (БЛА) [1, 2]. При этом основными тенденциями совершенствования средств воздушного нападения являются снижение их радиолокационной заметности за счет применения технологии Стелс при изготовлении и помеховое противодействие работе радиолокационных средств обнаружения [3-6].

Для решения задачи предупреждения о воздушном нападении и устранения несоответствия между требованиями к параметрам радиолокационного поля, составу и качеству ин- формации для обнаружения и сопровождения МВЦ и возможностями существующих средств их радиолокационного обнаружения и сопровождения целесообразно использовать перспективные радиолокационные средства, использующие при функционировании принципиально новые технические решения. Одним из таких направлений может быть разработка радиолокационных средств и комплексов обнаружения МВЦ на основе радиолокационной станциии (РЛС) дирижабельного базирования [7].

Одним из этапов создания дирижабельного радиолокационного комплекса (ДРЛК) обнаружения МВЦ и оценки эффективности его функционирования является разработка комплексной математической модели (КММ) данного комплекса. Такая модель включает следующие подэтапы: уяснение назначения и предполагаемого технического облика и особенностей при- 
менения ДРЛК; выбор показателей, характеризующих его боевые (информационные) возможности, и обоснование методов их оценки; формулирование требований к КММ и принципам ее построения; разработка структуры и обобщенных алгоритмов функционирования КММ ДРЛК; разработка алгоритмов функционирования отдельных подсистем ДРЛК и их математических (имитационных) моделей, реализуемых в КММ ДРЛК.

Частные вопросы разработки КММ при проектировании и создании сложных технических систем рассматриваются в [8-11]. Сложность и многообразие свойств и условий функционирования военной техники не позволяют описать их одной универсальной математической моделью. Кроме того, задачи оценки процесса функционирования военной техники являются комплексными и не могут быть сведены к простой арифметической сумме слабо увязанных между собой математических моделей и задач по расчету отдельных показателей.

Цель автора статьи - разработка КММ ДРЛК обнаружения МВЦ в интересах обоснования технического облика и особенностей применения ДРЛК, оценки эффективности его функционирования и боевых (информационных) возможностей по обнаружению различных типов МВЦ, обоснования требований к ДРЛК и принципам его построения и применения.

Разработка КММ ДРЛК обнаружения МВЦ основывается на основных принципах построения и структуре данного комплекса, являющегося информационным средством воздушно-космической обороны и представляющего собой сложную радиотехническую систему, аппаратно-программные средства которого должны размещаться как на земле - наземный пункт управления, приема и обработки информации (далеее - наземный пункт), так и в воздухе - на управляемой воздушной (дирижабельной) платформе.

Основным источником разведывательной информации ДРЛК является РЛС, размещаемая на дирижабле. Кроме этого, на дирижабле размещаются спецвычислитель управления комплексом и система его стабилизации. Важной составной частью бортовой аппаратуры является навигационная система, определяющая местоположение и ориентацию в пространстве ДРЛК и, соответственно, координаты точки его стояния, относительно которой выдается разведывательная информация. При этом управление перечисленными системами, а также орга- низацию их взаимодействия осуществляет бортовая система управления.

Информация об обнаруженных и сопровождаемых целях поступает на наземный пункт, который обеспечивает обмен данными пункта с бортовой частью ДРЛК, а также с вышестоящим командным пунктом воздушно-космической обороны; управление ДРЛК, в том числе режимами его работы, полетным заданием; отображение получаемой разведывательной информации.

На рисунке 1 приведена структура КММ ДРЛК, включающая несколько функционально законченных блоков и моделей: модель фоноиелевой обстановки (ФЦО), модель Земли, модель бортовых систем ДРЛК, включающую модель РЛС ДРЛК, модель бортовой системы управления ДРЛК и модель функционирования навигационной системы ДРЛК, модель наземного пункта, включающую модель отображения информации об обнаруженных и сопровождаемых целях ДРЛК и модель управления бортом, а также блок системы обработки и анализа результатов.

Модель ФЦО предназначена для имитации воздушной и помеховой обстановки, которая может складываться в пределах зоны обзора РЛС ДРЛК. Информация о реальных (эталонных) координатах моделируемых МВЦ поступает на модели бортовых систем ДРЛК (модели РЛС и др.), а также в систему обработки и анализа результатов моделирования, в которой данная информация используется в качестве эталонной для вычисления показателей качества траекторной информации: точности, полноты, достоверности. Модель Земли предназначена для учета взаимного пространственного расположения ДРЛК и земной поверхности с учетом наличия общей системы координат для моделируемых МВЦ и предполагает возможность учета рельефа земной поверхности и ее диэлектрических свойств. Модель бортовых систем ДРЛК включает модель бортовой системы управления, модель РЛС и модель навигационной системы. Управление моделями бортовых систем ДРЛК осуществляется посредством модели бортовой системы управления по командам, поступающим от наземного пункта. Команды могут содержать требования включить (выключить) ту или иную систему, изменить режим, изменить высоту или точку стояния ДРЛК.

С помощью модели РЛС ДРЛК осуществляется моделирование обнаружения МВЦ, имитируемых моделью ФЦО. Модель навигацион- 
ной системы предназначена для корректировки угловых координат обнаруженных целей и ДРЛК. Модель бортовой системы управления ДРЛК осуществляет объединение поступающей от моделей информации и выдает обобщенную информацию на модель отображения и в систему обработки и анализа результатов моделирования.

Рассмотрим более подробно функционирование блоков КММ ДРЛК.

Модель ФЦО включает в себя воздушнокосмическую целевую и радиоэлектронную обстановки. Под воздушно-космической целевой обстановкой понимаются пространственное положение и ориентация МВЦ относительно рассматриваемой точки. Радиоэлектронная обстановка в конкретной точке пространства моделируется совокупностью излучений бортовых радиоэлектронных средств МВЦ, находящихся в прямой видимости (в том числе с учетом рельефа местности) для рассматриваемой точки стояния ДРЛК. При этом сигналы излучаемых радиоэлектронных средств МВЦ характеризуются следующими параметрами: несущая частота, вид модуляции, период повторения, длительность импульсов и т.д., а также направлением их прихода и интенсивностью. Различают два варианта моделирования ФЦО - статический и динамический.
При статическом моделировании ФЦО задается отражательными (излучательными) характеристиками МВЦ в диапазонах длин волн информационных средств ДРЛК, а радиоэлектронная обстановка - статической совокупностью точек расположения помехопостановщиков и параметрами излучаемых ими помех. На основании этих данных оцениваются такие показатели, как зоны контроля и обнаружения РЛС ДРЛК МВЦ, параметры радиолокационного поля и др.

При динамическом моделировании ФЦО каждая МВЦ представляется совокупностью координат, характеризующих ориентацию цели при полете по некоторой траектории. При этом каждая цель характеризуется своими отражательными (излучательными) характеристиками в зависимости от ракурса ее наблюдения и характеристиками (параметрами) излучаемых ею помех в каждой точке траектории.

Для всесторонних исследований в КММ ДРЛК модель ФЦО в своем составе имеет модели (БД) средств воздушного нападения, к которым относятся аэродинамические, аэробаллистические и баллистические летательные аппараты всех классов, как существующие, так и перспективные. Обобщенный перечень необходимых для моделирования МВЦ в составе КММ ДРЛК следующий: пилотируемая авиа-

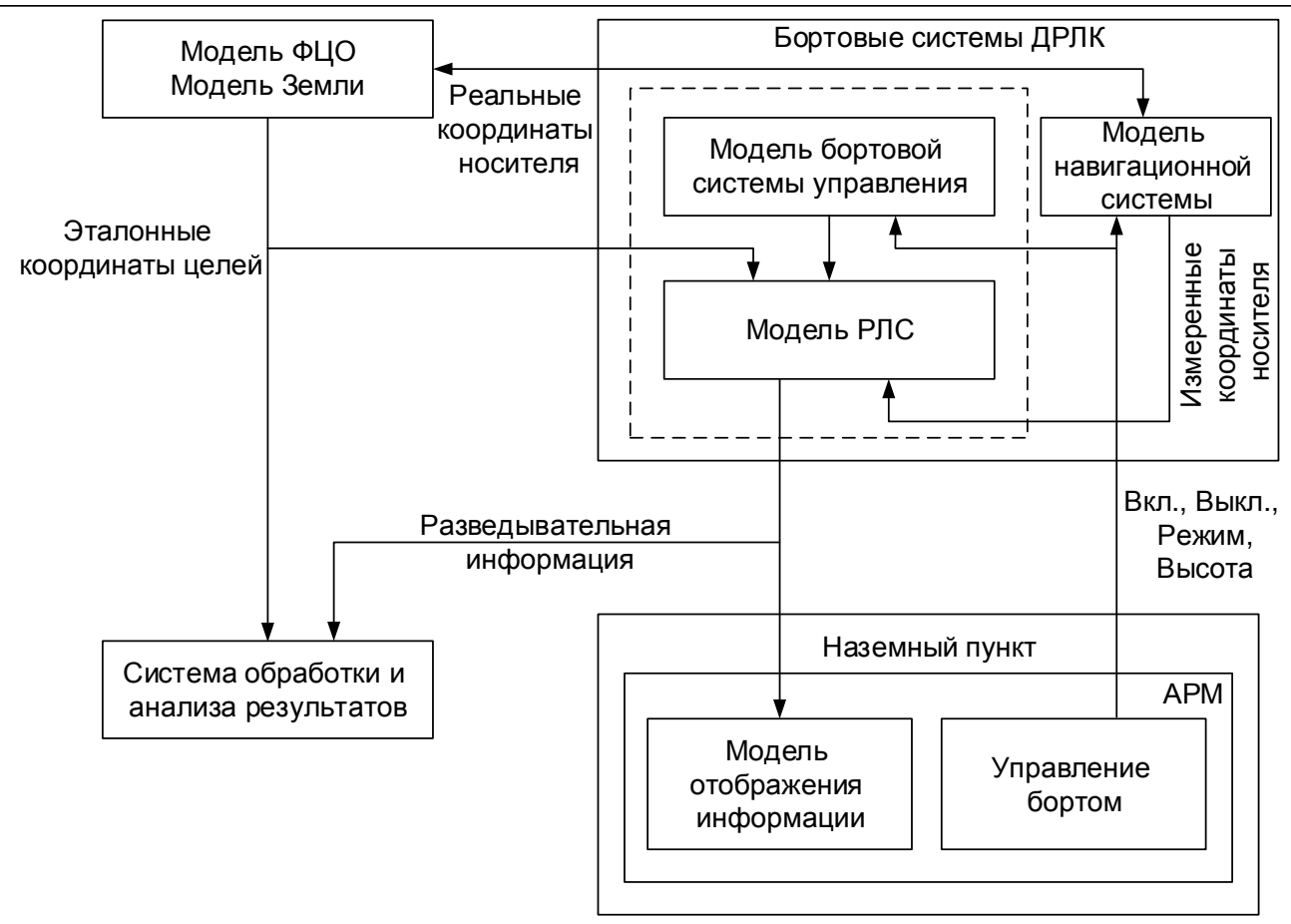

Рис. 1. Структура КММ ДРЛК

Fig. 1. The structure of the complex mathematical model of an airship radar system 
ция (самолеты, вертолеты), беспилотные средства, аэробаллистические средства. При этом имитация полета МВЦ проводится с учетом их летно-технических характеристик. Дополнительно при моделировании полета МВЦ в КММ возможно моделирование бортовых постановщиков активных помех.

В КММ ДРЛК моделирование ФЦО МВЦ сводится к трем составляющим: моделирование отражательных (излучательных) характеристик МВЦ, моделирование сигналов, излучаемых бортовыми радиоэлектронными средствами МВЦ, моделирование полета МВЦ.

Модель отражательных и излучательных характеристик МВЦ предназначена для расчета полезных сигналов при моделировании активных радиолокационных обнаружителей. В основе модели лежит статистическое описание эффективной площади рассеяния цели в виде параметрически заданного закона ее распределения. Данным параметром является среднее или медианное значение эффективной площади рассеяния. Закон распределения эффективной площади рассеяния цели (параметр закона распределения) при этом зависит от соотношения геометрических характеристик цели и длины волны, зондирующего сигнала при заданном ракурсе наблюдения и поляризации зондирующего сигнала. Для учета этих факторов модель отражательных характеристик МВЦ содержит ракурсные зависимости параметра и вида закона распределения эффективной площади рассеяния для заданных диапазонов длин волн и видов поляризации, в том числе с учетом сопутствующих полету МВЦ физических явлений, таких как факел двигателя, ударная волна, плазменные образования, спутный след и др.

В КММ ДРЛК моделирование процессов обнаружения МВЦ основывается на методах активной и пассивной радиолокации. При моделировании процессов обнаружения МВЦ методами активной радиолокации РЛС ДРЛК для расчета полезного сигнала используются данные об отражательных характеристиках обнаруживаемой МВЦ, а при расчете уровня помехи - о параметрах помех, излучаемых всеми МВЦ. При моделировании процессов обнаружения МВЦ средствами пассивной локации (пеленгация в радиолокационных диапазонах) для расчета уровней полезного сигнала используются данные об излучательных характеристиках, в том числе о сигналах, излучаемых бортовыми радиоэлектронными средствами МВЦ, а при расчете уровня помехи - данные о характеристиках и параметрах искусственных (сигналы, излучаемые остальными МВЦ) и естественных фоновых излучений.

Моделирование алгоритмов сопровождения МВЦ включает имитацию процесса их перемещения (полета). При этом требуемый уровень детализации модели полета МВЦ существенно зависит от характеристик и алгоритмов их сопровождения. В основе имитации полета МВЦ в представленной модели лежит динамика изменения их пространственного положения. Для задач функционирования ДРЛК (моделирование алгоритмов обнаружения, сопровождения и их комплексирования) учитываются два момента, которые определяют принципы динамического моделирования полета МВЦ.

Первым моментом является моделирование собственно движения МВЦ по заданному маршруту. Имитация движения МВЦ проводится в соответствии с заданным маршрутом (траекторией) ее движения в зависимости от текущего времени. Для этого МВЦ представляется в виде материальной точки и разделения модели полета аэродинамических и баллистических объектов. Необходимость разделения моделей связана с разными физическими принципами, на которых основан их полет, а также с существенными отличиями алгоритмов их сопровождения средствами ДРЛК.

Моделирование движения аэродинамических МВЦ основывается на алгоритмах их сопровождения, ориентированных на раздельное сопровождение по плоскостным координатам и высоте. Для этого МВЦ представляется в виде материальной точки, вектор состояния которой описывается тремя координатами и их первыми (вектором скорости) и вторыми (вектором ускорения) производными. При этом уравнение движения МВЦ описывается полиномом второй степени. При переходе к гипотезе движения, отличающейся от прямолинейного, используются алгоритмы обнаружения маневра, в основе которого лежит нелинейная модель движения цели, которая описывается кривой второго порядка. Поэтому для корректного моделирования маневрирующих по курсу и по высоте аэродинамических МВЦ используется сопряжение прямолинейных участков кривыми второго порядка, а параметры движения МВЦ на участках маневра (ускорения-перегрузки) не должны выходить за рамки летнотехнических характеристик МВЦ.

Формирование списка маневров проводится по следующим принципам: количество маневров должно быть минимальным; среднеквадра- 
тичное отклонение вертикального профиля маршрута МВЦ от эталонного должно быть минимальным; не допускается столкновение с землей, то есть профиль маршрута МВЦ не должен пересекаться с профилем рельефа местности. При этом общий алгоритм формирования списка маневров предусматривает последовательное применение данной процедуры для всех пар соседних точек профиля. Сформированный профиль проверяется на предмет столкновения с землей. Факт столкновения визуально отображается оператору, что означает невозможность прохода данного маршрута, и проводится новый расчет при менее жестких условиях на основе увеличения высоты изменения движения МВЦ.

Моделирование движения баллистических целей проводится на отельных моделях заранее, а в КММ ДРЛК используется метод межузловой интерполяции заранее рассчитанной таблицы развертки траектории их движения по времени.

Вторым моментом динамического моделирования полета МВЦ является имитация процессов функционирования их бортовых радиоэлектронных средств. В общем случае основными причинами, влияющими на радиоэлектронную обстановку в некоторой точке, являются дальность до МВЦ и изменение характеристик диаграммы направленности антенны бортовых радиоэлектронных средств МВЦ в процессе полета.

Модель Земли предназначена для учета взаимного пространственного расположения ДРЛК и земной поверхности. При этом математическое моделирование предполагает наличие общей системы координат для всех моделируемых МВЦ и ДРЛК с учетом рельефа земной поверхности и ее диэлектрических свойств. В КММ ДРЛК положение точки на поверхности Земли и в пространстве вокруг нее (в достаточно большом радиусе) задается в таких системах координат, как геоцентрическая и геодезическая. КММ ДРЛК позволяет осуществлять преобразование из геодезической системы координат в геоцентрическую систему координат. Геодезическая и геоцентрическая системы координат используются для задания местоположения всех моделируемых МВЦ и ДРЛК соответственно. В этом случае обеспечивается однозначное определение взаимного расположения объектов относительно друг друга.

В представленной КММ ДРЛК в модели РЛС используются измерения координат целей в сферической системе координат (дальность, азимут, угол места), а при обработке и передаче радиолокационной информации используются следующие системы: местная топоцентрическая система координат; местная топоцентрическая система плоскостных координат и высота относительно уровня моря. При этом используемая информационными датчиками РЛС ДРЛК для измерения координат МВЦ сферическая система координат пересчитывается в топоцентрическую систему координат, что определяет удобство топоцентрической системы координат для данных применений в КММ ДРЛК.

Для моделирования обнаружения МВЦ в качестве единой системы координат для наземного пункта используется местная топоцентрическая система плоскостных координат с высотой относительно точки стояния наземного пункта. Применение такой системы координат наиболее удобно для отображения трассовой информации, решения задач оценки воздушной обстановки и целераспределения. Координаты целей в трассовой информации, поступающей от ДРЛК, пересчитываются в единую систему координат наземного пункта. Последовательность действий состоит в следующем. ДРЛК передает собственные координаты (получаемые от навигационной системы) на наземный пункт в геодезическую систему координат. Координаты наземного пункта считаются известными. Затем осуществляется пересчет из местной топоцентрической системы координат в систему координат наземного пункта, а следом - расчет координат МВЦ в систему координат наземного пункта. При этом, если ДРЛК выдает координаты в местной топоцентрической системе координат с высотой, то первоначально вычисляются координаты высоты, а затем проводится их пересчет в систему координат наземного пункта. В том случае, если ДРЛК выдает координаты целей относительно наземного пункта, преобразование координат заключается во внесении поправки по координате высоты МВЦ с учетом точки стояния наземного пункта.

Обобщенная структурная схема модели РЛС ДРЛК приведена на рисунке 2. Модель строится на основе расчетов параметра обнаружения (отношения сигнал/шум) по уравнению радиолокации, являющемуся аналитической моделью приемо-передающего тракта и процесса распространения сигналов. Основными требованиями к математической (имитационной) модели РЛС ДРЛК являются следующие: 


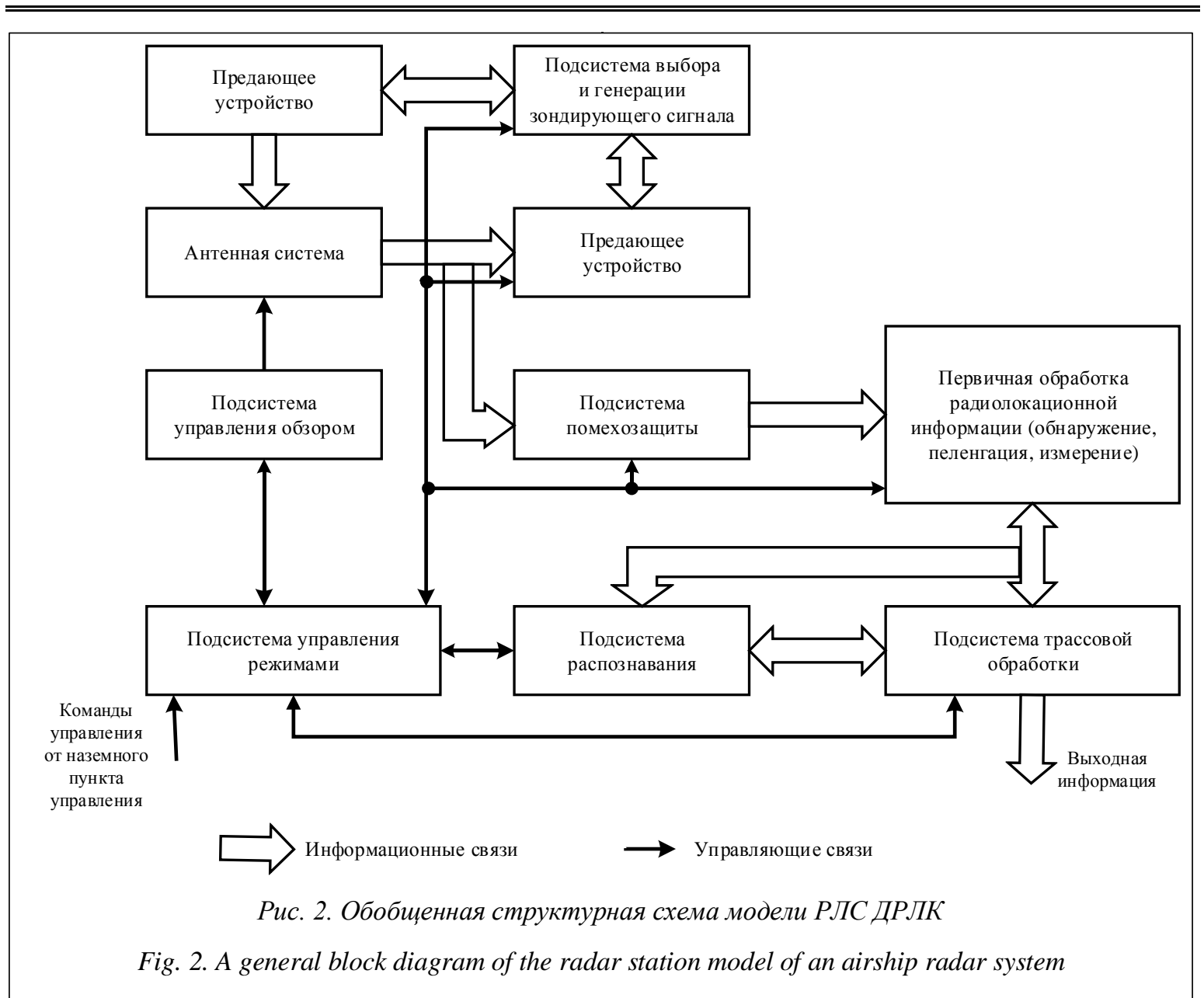

динамика функционирования РЛС ДРЛК имитируется путем последовательного изменения ее состояния через некоторые интервалы времени (шаг моделирования); модель обеспечивает динамический подыгрыш выходной информации, аналогичной выходной информации моделируемого средства (формуляры первичной или трассовой информации); модель предусматривает возможность имитации обмена с системой управления наземного пункта (прием управляющих команд и выдачу соответствующих квитанций о результатах их выполнения); модель имеет параметры, управление которыми позволит имитировать управление режимами функционирования моделируемого средства (режимами работы, работой подсистем и т.д.).

Математическая модель РЛС в КММ строится на основе РЛС кругового обзора, в которой передающие и приемные диаграммы направленности антенны формируются в одной антенной системе, обзор по азимуту осуществляется путем равномерного механического вращения антенны с периодом $T_{\text {об̆з, }}$ a обзор по углу места реализуется двумя способами: с помощью одного или нескольких неуправляемых разнесенных по углу места и одновременно функционирующих приемных парциальных каналов; электронным сканированием диаграммы направленности антенны по углу места по детерминированной программе. Характерными особенностями модели РЛС кругового обзора в КММ ДРЛК являются следующие: выраженная направленность диаграммы направленности антенны в азимутальной плоскости (ширина луча диаграммы направленности антенны $-\Theta_{\Gamma л}<<2 \pi$; уровни боковых лепестков и фона $-g_{\text {Бл, }} g_{\text {Фн }}<<1$; в вероятность обнаружения эхо-сигналов мала и при моделировании не учитывается; при моделировании не учитывается амплитудная модуляция принимаемых сигналов, вызванная вращением диаграммы направленности антенны.

Структуру модели РЛС в КММ ДРЛК определяют следующие основные режимы.

1. РЛС ДРЛК осуществляет круговой обзор пространства с темпом 5 или 10 с в соответствии с назначенным режимом обзора. Для об- 
наружения баллистических целей и гиперзвуковых летательных аппаратов предусматриваются специальные режимы секторного обзора.

2. Обнаружение МВЦ осуществляется в амплитудном или когерентном режиме работы РЛС ДРЛК. Причем когерентный режим назначается для обнаружения маловысотных целей на фоне интенсивных отражений от подстилающей поверхности Земли при круговом обзоре пространства с темпом $10 \mathrm{c}$.

3. Организация заданного обзора (секторного (кругового обзора)) осуществляется в соответствии со специальными алгоритмами управления для обнаружения конкретных МВЦ.

Модель управления ДРЛК. Основным предназначением данной модели является управление работой модели РЛС ДРЛК (подключение тех или иных ветвей алгоритмов модели функционирования) в соответствии с заданными режимами. Особенностью алгоритма управления режимами работы имитационных моделей РЛС ДРЛК является возможность поочередного включения режимов обзора РЛС. Когерентный режим может быть включен при темпе обзора 10 с, амплитудный - при любом режиме обзора. При этом первичная радиолокационная информация с выхода рассматриваемой модели поступает на вторичную обработку информации.

Модель отображсения информациі об обнаруженных и сопровожсдаемых целях ДРЛК. В соответствии со структурой КММ ДРЛК модель отображения информации обеспечивает следующее: отображение информации об обнаруженных (сопровождаемых) РЛС ДРЛК целях в процессе функционирования модели в удобном для восприятия и анализа виде; отображение (индикацию) информации о текущем режиме функционирования модели РЛС ДРЛК; возможность управления режимами функционирования модели РЛС (интерфейс управления). Учитывая специфическую структуру всего моделируемого объекта (воздушную и наземную части комплекса), дополнительно обеспечивается отображение информации о целях на выходе как системы обработки информации модели РЛС ДРЛК (воздушная часть комплекса), так и системы обработки информации наземного пункта (трассовой информации). Модель отображения информации включает следующие основные элементы: индикатор кругового обзора, предназначенный для отображения информации с выхода модели системы первичной обработки РЛС ДРЛК в ко- ординатах «азимут-дальность»; индикатор кругового обзора, предназначенный для отображения информации с выхода модели системы первичной обработки РЛС ДРЛК в координатах «азимут-угол места»; табличный индикатор информации о целях (формуляров целей); индикатор отображения воздушной обстановки наземного пункта; панель управления основными режимами работы модели РЛС ДРЛК.

Модель функционирования навигационной системы ДРЛК использует исходные данные положения носителя: вектор ошибок определения местоположения носителя - начала местной топоцентрической системы координат, связанной с носителем (например, фазовым центром антенны РЛС ДРЛК), и вектор ошибок определения ориентации осей местной топоцентрической системы координат, определяющий ошибки определения углов крена, тангажа и рысканья (соответственно $\alpha, \beta, \gamma)$ для связанной с носителем системы координат. Значения данных векторов ошибок определяются тактико-техническими характеристиками навигационной системы и задаются как исходные данные при моделировании. Моделирование функционирования навигационной системы осуществляется следующим образом. К заданным в начале моделирования истинным координатам положения носителя в каждом цикле, моделирующем функционирование навигационной системы, добавляется случайный вектор, элементами которого являются реализации независимых случайных величин, распределенных по нормальному закону со средними значениями и с дисперсиями, определяемыми вектором ошибок. Ошибки определения ориентации осей моделируются случайным вектором, элементами которого являются реализации независимых случайных величин, распределенных по нормальному закону с нулевым средним и с дисперсиями, определяемыми вектором ошибок. При этом вычисленные для исходной местной топоцентрической системы координат на основе углов крена, тангажа и рысканья $(\alpha=0, \beta=0, \gamma=0)$ прямоугольные координаты обнаруженной цели (вектор $\left.\mathbf{r}_{ц}=\left(x_{ц}, y_{ц}, z_{ц}\right)^{T}\right)$ корректируются в соответствии с выражением $\mathbf{r}_{\text {выд. }}=\mathbf{A}^{T} \mathbf{r}_{ц}$, где $\mathbf{A}-$ ортогональная матрица, элементы которой определяются через углы поворота. Поскольку матрица $\mathbf{A}$ есть композиция трех поворотов относительно осей координат OX, OY, OZ на углы $\alpha, \beta, \gamma$ соответственно, то при моделировании она представляется в виде произведения трех матриц поворотов. 


\section{Выводы}

Разработанная КММ ДРЛК обнаружения МВЦ используется для обоснования технического облика ДРЛК и особенностей его применения. Результаты моделирования функционирования ДРЛК на КММ позволяют оценить эффективность функционирования ДРЛК и его боевые (информационные) возможности по обнаружению различных типов МВЦ, обосновать методы их оценки, сформулировать требования к ДРЛК и принципам его построения, разработать структуру и обобщенные алгоритмы функционирования ДРЛК и его подсистем.

\section{Лuтература}

1. Лопин Г., Ваганов А., Миллер А., Смирнов М., Храмичев А. Оружие нового века // Воздушно-космический рубеж. 2018. № 3. С. 8-13.

2. Ломов Л. Оружие первого дня войны // Воздушно-космический рубеж. 2017. № 4. C. $44-50$.

3. Помехозащита радиоэлектронных систем управления летательными аппаратами и оружием; [под ред. В.Н. Лепина]. М.: Радиотехника, 2017. 416 с.

4. Радиоэлектронное подавление информационных каналов систем управления оружием; [под ред. Ю.М. Перунова]. М.: Радиотехника, 2003. $416 \mathrm{c}$.

5. Филин С.А., Молохина Л.А. Средства снижения заметности (по патентным материалам). М.: ИНИЦ Роспатента, 2003. 214 с.

6. Алексеев А.Г., Штагер Е.А., Козырев С.В.
Физические основы технологии Stealth. СПб: Изд-во ВВМ, 2007. 284 с.

7. Суша С.В. Обоснование необходимости применения дирижабельного радиолокационного комплекса дальнего обнаружения малозаметных воздушных целей // Передача, прием, обработка и отображение информации о быстропротекающих процессах: сб. матер. XXIX Всерос. науч.-технич. конф. школы-семинара. М.: Изд-во Акад. Жуковского, 2018. С. 29-34.

8. Созинов П.А. Актуальные задачи математического моделирования систем воздушно-космической обороны // Вестн. Концерна ВКО «Алмаз - Антей». 2017. № 3. С. 17-26.

9. Боев С.Ф. Концептуальная основа системы автоматизированного эскизно-технического проектирования перспективных РЛС дальнего обнаружения // Вестн. компьютер. и информ. технологий. 2014. № 8. DOI: 10.14489/vkit.2014.08.pp.003010 .

10. Ковалев Ф.Н., Хранилов В.П. Идентификация модели просветного радара на разных этапах технического проектирования радиолокационной системы // Cloud of Science. 2014. T. 1. № 3. URL: https://cloudofscience.ru/sites/default/files/pdf/CoS 1_383.pdf (дата обращения: 20.12.2018).

11.Груздов В.В., Леухин А.Н., Слока В.К. Управление процессами создания радиолокационных комплексов космического базирования на основе анализа отношения правдоподобия корреляционно-экстремальных функций полученной и синтезированной радиоголограмм // Журн. радиоэлектроники. 2018. № 2. URL: http://jre.cplire.ru/jre/feb18/7/text.pdf (дата обращения: 20.12.2018).

\title{
A complex mathematical model of the air anti-stealth airship radar system
}

\author{
S.V.Susha ${ }^{1}$, Head of Department, sushasv2008@yandex.ru \\ ${ }^{1}$ Department of Ministry of Defense of Russia, Tver, 170100, Russian Federation
}

\footnotetext{
Abstract. One of the stages of creating an anti-stealth airship radar system and evaluating its effectiveness is the development of an integrated mathematical model. This paper presents the structure of such model. The model is developed to substantiate the technical appearance of the system and its application features, as well as to evaluate operating effect and combat (information) capabilities. A mathematical model of the system is based on the basic construction principles and a complex structure. The system itself is an information tool representing a complex radio system. The hardware and software of the system should be located both on the ground and in the air.

The structure of the system mathematical model includes several functionally complete blocks and models, in particular, models of a target environment, the Earth, on-board systems of the airship radar system, etc.
} 

action.

The paper describes particular mathematical models that are a part of an integrated one, their mutual inter-

The radar model of the dirigible radar system is based on calculating detection parameters (signal-noise ratio) using the radar equation. It is an analytical model of the receiving-transmitting path and a signal propagation process. Successive changing of its state at certain time intervals simulates the functioning dynamics. The model provides a dynamic playout of the input information to the similar output information of a simulated tool. In addition, it provides the ability to simulate an exchange with a control system, has parameters that allow simulating the control of simulated device operation modes.

The paper considers the possibilities of a complex mathematical model of an anti-stealth airship radar system.

Keywords: complex mathematical model, stealth air target, radar station, airship radar system.

\section{References}

1. Lopin G., Vaganov A., Miller A., Smirnov M., Khramichev A. Weapons of the new century. Airspace Line. 2018, no. 3, pp. 8-13 (in Russ.).

2. Lomov L. Weapons of the first day of the war. Airspace Line. 2017, no. 4, pp. 44-50 (in Russ.).

3. Interference Protection of Electronic Control Systems of Aircraft and Weapons. V.N. Lepin (Ed.). Moscow, Radiotekhnika Publ., 2017, 416 p.

4. Electronic Suppression of Information Channels of Weapon Control Systems. Yu.M. Perunov (Ed.). Moscow, Radiotekhnika Publ., 2003, 416 p.

5. Filin S.A., Molokhina L.A. Radar-Eluding Technology (Patent Materials). Moscow, INITs Rospatenta Publ., 2003, 214 p.

6. Alexeev A.G., Shtager E.A., Kozurev S.V. Physical Foundations of the Stealth Technology. St. Petersburg, VVM Publ., 2007, 284 p.

7. Susha S.V. A rationale for using anti-stealth airship advanced radar system. Transmission, Reception, Processing and Display of Information on Fast Processes: Proc. 29th All-Russ. Sci. and Tech. Conf. of a School Workshop. Moscow, Zhukovsky Academy Publ., 2018, pp. 29-34.

8. Sozinov P.A. Crucial tasks of mathematical modeling of aerospace defense systems. Bulletin of Concern VKO “Almaz-Antey”. 2017, no. 3, pp. 17-26 (in Russ.).

9. Boev S.F. The conceptual basis of the automated technical sketch design of perspective far detection radar stations. Herald of Computer and Information Technologies. 2014, no. 8, pp. 003-010 (in Russ.). DOI: 10.14489/vkit.2014.08.pp.003-010.

10. Kovalev F.N., Khranilov V.P. Identification of a transmission radar model at different stages of radar system technical design. Cloud of Science. 2014, vol. 1, no. 3. Available at: https://cloudofscience.ru/sites/default/files/pdf/CoS_1_383.pdf (accessed December 20, 2018).

11. Gruzdov V.V., Leukhin A.N., Sloka V.K. Management of the processes of creation of space-based radar complexes based on the analysis of the likelihood ratio of the correlation-extreme functions of the obtained and synthesized radio holograms. J. of Radio Electronics. 2018, no. 2. Available at: http://jre.cplire.ru/jre/ feb18/7/text.pdf (accessed December 20, 2018).

\section{Для цитирования}

Суша С.В. Комплексная математическая модель дирижабельного радиолокационного комплекса обнаружения малозаметных воздушных целей // Программные продукты и системы. 2019. Т. 32. № 2. C. 290-298. DOI: $10.15827 / 0236-235 X .126 .290-298$.

\section{For citation}

Susha S.V. A complex mathematical model of the air anti-stealth airship radar system. Software \& Systems. 2019, vol. 32 , no. 2 , pp. 290-298 (in Russ.). DOI: 10.15827/0236-235X.126.290-298. 